\title{
Addition polymerization of 1,1-dimesitylneopentylgermene: synthesis of a polygermene $\dagger$
}

\author{
Laura C. Pavelka, ${ }^{a}$ Simon J. Holder ${ }^{b}$ and Kim M. Baines* ${ }^{a}$ \\ Received (in Berkeley, CA, USA) 31st January 2008, Accepted 11th March 2008 \\ First published as an Advance Article on the web 17th April 2008 \\ DOI: $10.1039 / \mathbf{b 8 0 1 7 6 2 j}$
}

A new polymer with an alternating germanium-carbon backbone has been synthesized from 1,1-dimesitylneopentylgermene via addition polymerization using an anionic initiator.

Addition polymerization of vinylic monomers, a standard protocol for the preparation of organic polymers, has only recently been applied to the synthesis of inorganic polymers. The development of this important method for inorganicbased polymers was undoubtedly delayed by the lack of or difficulty in the synthesis of suitable monomers. In fact, much of the research on unsaturated inorganic, primarily main group, compounds over the last 30 years has focused on understanding how to prevent oligomerization reactions. One key strategy involves the use of bulky substituents to kinetically stabilize the doubly-bonded species; ${ }^{1}$ it follows that such compounds would not be suitable as monomers for polymer synthesis. However, in a landmark series of papers, Gates et al. have shown that readily accessible stable phosphaalkenes $(\mathrm{P}=\mathrm{C})$ with relatively bulky substituents can indeed undergo addition polymerization using radical or anionic initiators to form poly(methylenephosphine)s, a new and interesting class of polymers. ${ }^{2}$ Furthermore, the phosphaalkenes undergo living anionic polymerization at ambient temperatures using organolithium reagents as initiators. ${ }^{3}$ This discovery has opened up numerous exciting possibilities for the synthesis of novel copolymers containing the functional poly(methylenephosphine) block. Block copolymers, in general, and particularly those with inorganic segments, have been the subject of intense research of late due to the spontaneous self assembly of the polymers into varied and interesting nanostructures. ${ }^{4}$

We have long been interested in the chemistry of multiplybonded germanium derivatives and were intrigued by the possibility that germenes $(\mathrm{Ge}=\mathrm{C})$ may also be able to undergo addition polymerization to provide an entry into a hitherto unknown $[\mathrm{GeC}]_{n}$ polycarbogermane system. Given the extensive interest in poly(silylenemethylene) polymers, $[\mathrm{SiC}]_{n},{ }^{5}$ particularly in their pyrolysis chemistry to give silicon carbide, ${ }^{6}$

\footnotetext{
${ }^{a}$ Department of Chemistry, University of Western Ontario, 1151

Richmond Street, London, Ontario, Canada N6A 5B7. E-mail:

kbaines2@uwo.ca; Fax: +1 (519) 661-3022;Tel: +1 (519) 661-

2111

${ }^{b}$ Functional Materials Group, School of Physical Sciences, University of Kent, Canterbury, UK CT2 $7 \mathrm{NH}$

$\dagger$ Electronic supplementary information (ESI) available: Experimental procedures, ${ }^{1} \mathrm{H}$ and ${ }^{13} \mathrm{C}$ NMR spectra, TGA data, DSC data, GPC data and crystallographic data for CCDC 676604 in CIF format. See DOI: $10.1039 / \mathrm{b} 801762 \mathrm{j}$
}

and the relatively scarce information available on the pyrolysis of polycarbogermanes, ${ }^{7}$ we have now investigated the addition polymerization of a solution stable germene as a new route to polymers with a $[\mathrm{GeC}]_{n}$ backbone.

A pale yellow pentane solution of 1,1-dimesitylneopentylgermene (1) was prepared from the addition of $t$-butyllithium (1 equiv.) to a solution of fluorovinylgermane $2 .{ }^{8}$ Germene $\mathbf{1}$ was stable in solution for several hours; however, upon addition of $t$-butyllithium ( 0.1 equiv.) to the solution (Scheme 1$)$, the colour of the solution changed from pale to bright yellow. After $30 \mathrm{~min}$, the reaction was quenched with methanol and the bright yellow colour dissipated immediately. The solvents were removed and the residue was dissolved in $\mathrm{CH}_{2} \mathrm{Cl}_{2}$. A white solid precipitated from the $\mathrm{CH}_{2} \mathrm{Cl}_{2}$ solution upon the addition of methanol; the solid was purified by re-precipitation. The air stable material (3) was isolated in $45 \%$ yield. The ${ }^{1} \mathrm{H}$ NMR spectrum of the solid displayed broad resonances consistent with a polymeric substance. No resonances attributable to the methanol adduct of germene $\mathbf{1}^{8}$ were observed in the residue or the precipitated material, indicating that all of the germene had been consumed prior to the addition of methanol. The rapid polymerization of $\mathbf{1}$ is in contrast to the much slower polymerization of phosphaalkenes. ${ }^{2,3}$ The molecular weight of the polymer was determined by GPC in THF; two distinct fractions were observed indicating a bimodal molecular weight distribution. The number-average molecular weights $\left(M_{\mathrm{n}}\right)$ were estimated to be $36000 \mathrm{~g} \mathrm{~mol}^{-1}$ and $7100 \mathrm{~g}$ $\mathrm{mol}^{-1}$ (vs. polystyrene) with polydispersity indices $(\mathrm{PDI}=$ $M_{\mathrm{w}} / M_{\mathrm{n}}$ ) of 1.5 and 1.1 , respectively. The bimodal molecular weight distribution was reproducible; similar $M_{\mathrm{n}}$ values were



Scheme 1 Synthesis of polygermene 3 . 
achieved. Polymer 3 was determined to be amorphous in nature based on the DSC results where only a glass transition was observed $\left(T_{\mathrm{g}}=144{ }^{\circ} \mathrm{C}\right)$. The thermal stability of polymer 3 was determined using TGA. The material was stable to weight loss until $290{ }^{\circ} \mathrm{C}$, at which point $87 \%$ of the mass was lost.

Polymer 3 can also be synthesized in one step from the addition of excess $t$-butyllithium (1.1 equiv.) to fluorovinylgermane 2 in pentane at $-78^{\circ} \mathrm{C}$. Upon warming, germene $\mathbf{1}$ formed quantitatively as noted by the pale yellow colour of the solution. When the solution reached room temperature, germene 1 then reacted with the remainder of the $t$-butyllithium, as indicated when the colour of the solution changed from pale to bright yellow. Quantitative formation of germene 1 under these conditions was verified in a separate experiment in which methanol was added to the solution immediately upon reaching room temperature. The methanol adduct of 1 was the only product observed.

After precipitation, the polymer was obtained in $62 \%$ yield. The number-average molecular weight $\left(M_{\mathrm{n}}\right)$ of the polymer prepared by this method was higher than that prepared previously $\left(M_{\mathrm{n}}=39000 \mathrm{~g} \mathrm{~mol}^{-1}\right.$, PDI $\left.=2.0\right)$. The broad PDI is possibly a result of initiation over an extended time period during the start of the polymerization resulting from the gradual warming of the solution. The addition of one portion of $t$-butyllithium is the preferred method for the formation of polymer 3 since monomodal higher molecular weight polymer was isolated in greater yield with fewer experimental manipulations.

The polymeric material (3) was characterized by one- and two-dimensional NMR spectroscopy. The ${ }^{13} \mathrm{C}$ NMR spectrum of $\mathbf{3}$ was particularly useful in elucidating the structure of the polymer. The signals in the ${ }^{13} \mathrm{C} N M R$ spectrum of $\mathbf{3}$ were readily assigned by comparison of the chemical shifts with those of the model compound, $\mathrm{Mes}_{2} \mathrm{Ge}(t \mathrm{Bu}) \mathrm{CH}_{2} \mathrm{CH}_{2} t \mathrm{Bu}^{8}$ For example, the $\mathrm{GeCH}$ and $\mathrm{GeCHCH}_{2}$ carbons in polymer 3 were observed to resonate at 16.3 and $38.7 \mathrm{ppm}$, respectively, compared to 14.4 and 40.5 ppm in the model compound. Similarly, signals attributable to the $\left(\mathrm{C}\left(\mathrm{CH}_{3}\right)_{3}\right)$ moiety, the ortho- and para-methyls and the aromatic carbons of the mesityl groups could be assigned. However, in the polymer, two sets of mesityl signals are observed resulting from meso and racemic replacements between the stereogenic carbon centres. The assignments were confirmed by both $2 \mathrm{D}{ }^{13} \mathrm{C}-{ }^{1} \mathrm{H}$ correlation spectroscopy and DEPT spectroscopy. There were no remaining, unassigned signals in the ${ }^{13} \mathrm{C}$ NMR spectrum of $\mathbf{3}$, indicating a regular alternating $[\mathrm{GeC}]_{n}$ backbone.

The regioselective addition of $t$-butyllithium to germene $\mathbf{1}$ dissolved in ether has been reported; upon quenching with water, only germane, $\mathrm{Mes}_{2} \mathrm{Ge}(t \mathrm{Bu}) \mathrm{CH}_{2} \mathrm{CH}_{2} t \mathrm{Bu}$, was isolated. ${ }^{8}$ Presumably, the polymerization of $\mathbf{1}$ is initiated by the regioselective addition of $t$-butyllithium to the germene to give $\mathrm{Mes}_{2} \mathrm{Ge}(t \mathrm{Bu}) \mathrm{CH}(\mathrm{Li}) \mathrm{CH}_{2} t \mathrm{Bu}$; in pentane, the carbanion rapidly adds to the germanium centre of another germene (1) to propagate the growth of the polymer. The decreased stability of the germene, along with a less sterically hindered propagating carbanion, likely contribute to the high reactivity. The bimodal distribution of polymer 3 synthesized by the addition
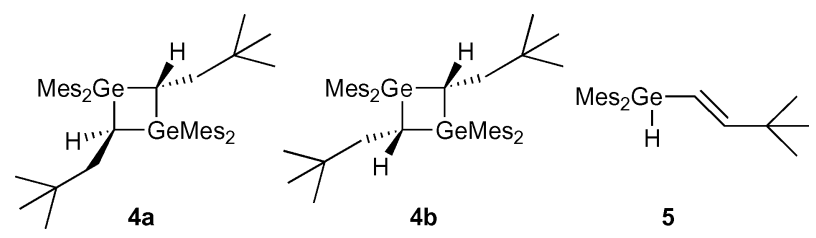

Fig. 1 Cyclic dimers (4a,b) and isomeric germane (5) of germene $\mathbf{1}$.

of 0.1 equiv. $t$-BuLi to the germene is indicative of more than one polymerization mechanism. Other possible concurrent mechanisms may include radical polymerization by electron transfer to $\mathbf{1}$ or even ring-opening polymerization involving the intermediate formation of germene dimers (4a and $\mathbf{4 b}$, vide infra). $\ddagger$

Germene 1 slowly reacted to give head-to-tail cyclic dimers $\mathbf{4 a}$ and $\mathbf{b}$, an isomeric germane (5), and polymeric material (Fig. 1) when left in solution $\left(\mathrm{C}_{6} \mathrm{D}_{6}\right)$ for several days. The head-to-head cyclic dimer was not observed. The two head-totail cyclic dimers $(\mathbf{4 a}, \mathbf{b})$ were consistently formed in a $1: 1$ ratio. However, the relative ratio of $\mathbf{4 a}$ and $\mathbf{b}$ to germane $\mathbf{5}$, presumably formed via a hydride shift, varied depending on the concentration of the germene. Germane $\mathbf{5}$ was favoured when the initial solution was dilute, whereas in concentrated solutions the cyclic dimers $(\mathbf{4 a}, \mathbf{b})$ were formed almost exclusively. The trans dimer (4a) selectively crystallized from a concentrated $\mathrm{C}_{6} \mathrm{D}_{6}$ solution. The molecular structure of $\mathbf{4 a}$ was determined by X-ray crystallography (Fig. 2). The 1,3digermacyclobutane ring lies in a plane. The intracyclic bond angles are close to $90^{\circ}$ and the intracyclic $\mathrm{Ge}-\mathrm{C}$ bond lengths, 2.016(2) and 2.041(2) $\AA$, are long but still well within the normal Ge-C single bond length range (1.90-2.05 $\AA$ ). ${ }^{9}$ The metrics of $\mathbf{4 a}$ are comparable to those of other crystallographically characterized 1,3-digermacyclobutanes. ${ }^{10}$ DSC analysis was performed on $\mathbf{4 a}$ and on a mixture of $\mathbf{4 a}-\mathbf{4 b}$. An irreversible endotherm was observed in each sample with an onset temperature of 290.4 and $248.7^{\circ} \mathrm{C}$, respectively. Despite

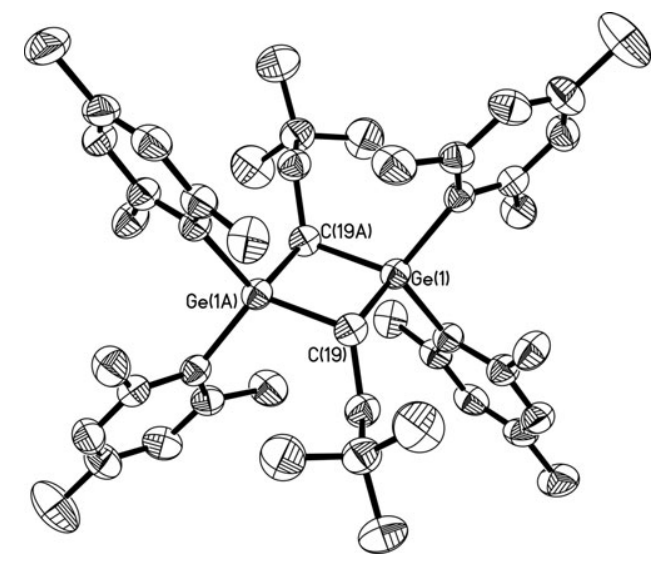

Fig. 2 Thermal ellipsoid plot (50\% probability surface) of 4 a. Two molecules of $\mathbf{4 a}$ are present in the asymmetric unit; parameters are given for one of the two molecules. Atoms labeled with 'A' are located at equivalent positions $(-x,-y,-z)$. Hydrogen atoms are omitted for clarity. Selected bond lengths $(\AA)$ and angles (deg): $\mathrm{Ge}(1)-\mathrm{C}(19)=$ 2.041(2), $\mathrm{Ge}(1)-\mathrm{C}(19 \mathrm{~A})=2.016(2), \quad \mathrm{C}(19)-\mathrm{Ge}(1)-\mathrm{C}(19 \mathrm{~A})=$ $90.11(10)$. 
the irreversibility, the endotherms appear to be melting transitions. In the case of $\mathbf{4 a}$, the endotherm coincides with the observed melting point $\left(280{ }^{\circ} \mathrm{C}\right)$, which was immediately followed by decomposition. No exotherm indicative of a thermal ring opening polymerization was observed.

The polymerization of germene $\mathbf{1}$ in the absence of an anionic initiator occured slowly; the polymer was formed as a minor component of the product mixture $(25 \mathrm{wt} \%)$ under these conditions. The polymer was isolated from the mixture by precipitation from $\mathrm{CH}_{2} \mathrm{Cl}_{2}$ with methanol. The ${ }^{1} \mathrm{H}$ and ${ }^{13} \mathrm{C}$ NMR spectra of the polymer obtained under these conditions were identical to the spectroscopic data of $\mathbf{3}$. The exclusive formation of head-to-tail cyclic dimers $(\mathbf{4 a}, \mathbf{b})$ under the same conditions was also taken as evidence for a polymer with an alternating $[\mathrm{GeC}]_{n}$ backbone. The molecular weight of the polymer was lower than that obtained using an anionic initiator and had a broader polydispersity $\left(M_{\mathrm{n}}=21000 \mathrm{~g} \mathrm{~mol}^{-1}\right.$ vs. polystyrene; PDI $\left.=2.5\right)$. This polymerization is presumably a result of a radical polymerization process, though further experiments are needed to verify this.

In summary, we have reported the first addition polymerization of a stable germene to yield a new inorganic polymer, a polygermene. We are currently exploring whether the polymerization can be carried out in a living fashion with the goal of synthesizing block copolymers containing this new inorganic segment.

We thank the Natural Sciences and Engineering Research Council of Canada, the University of Western Ontario, the Canadian Foundation for Innovation-Ontario Innovation Trust, and the Ontario Photonics Consortium for financial support. We also thank Teck Cominco Ltd. for a donation of $\mathrm{GeCl}_{4}$, D. Hairsine for acquisition of mass spectral data, Dr M. C. Jennings for acquisition of X-ray diffraction data, and the ASPIRE program (UWO; A Special International Research Experience) for the opportunity for L. C. P. to study at the University of Kent under the supervision of Dr Simon Holder.

\section{Notes and references}

$\ddagger$ Any significant termination process in an ionic polymerization typically leads to broader monomodal molecular weight distributions rather than a bimodal molecular weight distribution. ${ }^{11}$

1 For reviews see: (a) E. Rivard and P. P. Power, Inorg. Chem., 2007, 46, 10047; (b) M. Driess and H. Grützmacher, Angew. Chem., Int. Ed. Engl., 1996, 35, 828; (c) N. C. Norman, Polyhedron, 1993, 12, 2431; (d) A. H. Cowley, Acc. Chem. Res., 1984, 17, 386.

2 (a) C. W. Tsang, M. Yam and D. P. Gates, J. Am. Chem. Soc., 2003 , 125, 1480; (b) C. W. Tsang, B. Baharloo, D. Riendl, M. Yam and D. P. Gates, Angew. Chem., Int. Ed., 2004, 43, 5682; (c) K. J. T. Noonan, B. O. Patrick and D. P. Gates, Chem. Commun., 2007, 3658.

3 K. J. T. Noonan and D. P. Gates, Angew. Chem., Int. Ed., 2006, 45, 7271.

4 For reviews see: (a) J. E. Mark, H. R. Allcock and R. West, Inorganic Polymers, Oxford University Press, Oxford, 2005; (b) I. Manners, Angew. Chem., Int. Ed. Engl., 1996, 35, 1602; (c) I. Manners, Angew. Chem., Int. Ed., 2007, 46, 1565.

5 For reviews see: $(a)$ L. V. Interrante, Q. Liu, I. Rushkin and Q. Shen, J. Organomet. Chem., 1996, 521, 1; (b) L. V. Interrante, I. Rushkin and Q. Shen, Appl. Organomet. Chem., 1998, 12, 695; (c) W. Uhlig, in Organosilicon Chemistry IV: From Molecules to Materials, ed. N. Auner and J. Weis, Wiley-VCH, Weinheim, 2000, pp. 563; (d) W. Uhlig, Prog. Polym. Sci., 2002, 27, 255.

6 (a) M. Birot, J. P. Pillot and J. Dunogues, Chem. Rev., 1995, 95, 1443; (b) Q. Liu, H. J. Wu, R. Lewis, G. E. Maciel and L. V. Interrante, Chem. Mater., 1999, 11, 2038; (c) Q. M. Cheng, L. V. Interrante, M. Lienhard, Q. Shen and Z. Wu, J. Eur. Ceram. Soc., 2005, 25, 233.

7 J. L. Brefort, R. J. P. Corriu, D. Guerin and B. J. L. Henner, J. Organomet. Chem., 1994, 464, 133.

8 C. Couret, J. Escudié, G. Delpon-Lacaze and J. Satgé, Organometallics, 1992, 11, 3176.

9 K. M. Baines and W. G. Stibbs, Coord. Chem. Rev., 1995, 145, 157.

10 (a) A. I. Gusev, T. K. Gar, M. G. Los and N. V. Alexeev, J. Struct. Chem., 1976, 17, 736; (b) N. N. Zemlyanskii, I. V. Borisova, V. K. Bel'skii, N. D. Kolosova and I. P. Beletskaya, Izv. Akad. Nauk. SSSR, Ser. Khim., 1983, 959; (c) N. P. Toltl, M. Stradiotto, T. L. Morkin and W. J. Leigh, Organometallics, 1999, 18, 5643; (d) N. Wiberg, T. Passler, S. Wagner and K. Polborn, J. Organomet. Chem., 2000, 598, 292; (e) W. P. Leung, Z. X. Wang, H. W. Li, Q. C. Yang and T. C. W. Mak, J. Am. Chem. Soc., 2001, 123, 8123; $(f)$ W. P. Leung, K. W. Wong, Z. X. Wang and T. C. W. Mak, Organometallics, 2006, 25, 2037.

11 M. Szwarc, Carbanions, Living Polymers and Electron Transfer Processes, John Wiley and Sons, New York, 1969. 